\title{
PREVALANCE OF MALE INFERTILITY IN INDIA: STUDIES ON THE EFFECTS OF GONADOTROPIN RELEASING HORMONES
}

\author{
RADHAKRISHNAN DEVENTHIRAN ${ }^{1}$, KUMARESAN RAMANATHAN ${ }^{2}$, NAGAMURUGAN NANDAKUMAR ${ }^{3 *}$
}

${ }^{1}$ Department of Biotechnology, Kurinji College of Arts and Science, Tiruchirappalli, Tamil Nadu, India. ${ }^{2}$ Department of Biochemistry, Institute of Biomedical Science, College of Health Sciences, Mekelle University (Ayder Campus), Mekelle, Ethiopia. ${ }^{3}$ Department of Botany, Government Arts College, Melur, Madurai - 625 106, Tamil Nadu, India. Email: naga06@yahoo.com

Received: 03 March 2017, Revised and Accepted: 03 May 2017

ABSTRACT

Objective: Nowadays, there is an increased incidence of infertility in Indian males due to lifestyle changes. Hence, the objective of this study is evaluating the gonadotropin releasing hormones $(\mathrm{GnRH})$ level in infertile young male in Indian population.

Materials and Methods: In total, 56 patients having abnormal semen count and five control patients have been included in the study. All patients were underwent sperm count and estimation of hormones includes GnRH such as follicle stimulating hormone (FSH), tri-iodothyronine, thyroxin, prolactin, and testosterone.

Results: The sperm concentration of infertile men was significantly lower than control. Sperm motility behaviors rapid, sluggish, and non-motile characters were significantly lower than control. Among GnRH, FSH has been significantly higher in infertile group than control group.

Conclusion: FSH may be considered as a marker for male infertility.

Keywords: Infertility, Gonadotropin releasing hormones, Follicle stimulating hormone, Indian male, Biomarkers, Sperm count.

(C) 2017 The Authors. Published by Innovare Academic Sciences Pvt Ltd. This is an open access article under the CC BY license (http://creativecommons. org/licenses/by/4. 0/) DOI: http://dx.doi.org/10.22159/ajpcr.2017.v10i8.18254

\section{INTRODUCTION}

Infertility is a worldwide problem and it affects $15 \%$ of married couples worldwide [1]. The prevalence of infertility in the general population is $15-20 \%$, and of this, the male factor is responsible for $20-40 \%$ [2,3], and in India, the prevalence is around $23 \%$ [4]. In a preliminary study by the World Health Organization multi-center study, $45 \%$ of infertile men were found to be affected by oligozoospermia or azoospermia [5-7]. The semen analysis is initially assessed and if it is found abnormal (especially if sperm concentration is $<15$ million per milliliter) [8] then the sample is subjected for further analysis to ascertain the possibilities of hormonal disorders or imbalance. The patients with such abnormal sperm count are recommended to undertake a blood hormonal test as these hormonal tests are found to be reliable and accurate [9]. Men produce both, male hormones (testosterone) as well as pituitary hormones such as follicle stimulating hormone (FSH) and luteinizing hormone (LH) which are among the gonadotropin releasing hormones (GnRH) [10]. Blood tests for testosterone and FSH levels are usually taken initially and if the testosterone levels are low, then LH is measured [11]. Low levels of FSH, LH, and testosterone may indicate a diagnosis of hypogonadotropic hypogonadism [12,13], and a very high FSH levels (compared to normal levels of other hormones) indicate abnormalities in initial sperm production [10]. In India, the prevalence of infertility in men is increasing steadily and it has reached to such alarming levels that its incidence may affect the male population in future. The incidence cannot be ignored, therefore a detailed study involving the sperm count and hormonal profile especially FSH, LH, and testosterone concentration is an urgent necessity to ascertain the fertility problems in men [14]. Unfortunately, this study is poorly documented in India; therefore, this study can be considered as a step toward evaluating the GnRH level in infertile young male in Indian population.

\section{METHODS}

The study involved analysis of primary health records of around 56 patients (aged above 32) having abnormal semen count. The sample also included 5 controls comprising healthy volunteers. This study has been approved by hospital ethics committee. The clinical examination and the lifestyle of each individual were collected which comprised their age, health problems, food habit, duration of marriage, and history of infertility in the family. The semen samples were collected from the infertile subjects after a prerequisite of 2-7 days of sexual abstinence. Estimation of sperm counting was done using the Meckler's chamber, and morphology study was based on the smear diff quick stain method. Sperm analysis was performed according to the World Health Organization guidelines. Based on the sperm concentration, the infertile subjects were classified into normozoospermia ( $>15$ million sperm $/ \mathrm{ml}$ ), oligoasthenozoospermia $A+B(\geq 32$ million sperm $/ \mathrm{ml}$ ), and azoospermia (no spermatozoa) wherein in fertile controls, the sperm count ranged from 15 to 120 million sperms $/ \mathrm{ml}$. The scrotal Doppler test was done and the grade of varicoceles was recorded. Testicular biopsy was carried out to understand the histopathological condition of the samples. The serum tri-iodothyronine (T3) and thyroxin (T4), thyroid stimulating hormone (TSH), LH, FSH, Prolactin, and Testosterone levels in all the samples including control were collected and analyzed. The hormonal assays was done by Chemi luminescence method using the kits supplied by Diagnostic Products Corporation; USA. The data were analyzed using Medcalc statistical software. The mean t-test was done to understand the significance of the study.

\section{RESULTS AND DISCUSSION}

The mean sperm concentration in infertile males $(33.52 \pm 30.50)$ was found to be very low compared to fertile controls $(73.7 \pm 36.39)$ which relates to its abnormal nature. Among the data, the values having mean t-test with p value $(<0.05)$ were considered for analysis. Here, it is noted that the sperm concentration is directly proportional to the high value of normal counts compared to the abnormal counts so that we can easily predict the sperm concentration based on the given statistical data in the study (Fig. 1). 
Observation of the sperm motility behaviors such as rapid (A), sluggish (B), twitching (C), and non-motile (D) in infertile males showed that there was a significant decrease in rapid $(5.73 \pm 4.90$; normal: $10.0 \pm 5.45)$, sluggish (29.64 \pm 14.76 ; normal: $40.4 \pm 6.8$ ) and non-motility (52.41 \pm 17.92 ; normal: $38.1 \pm 8.96$ ) behavior, but there was insignificant changes in twitching $(12.20 \pm 6.97$; normal: $11.5 \pm 4.6)$ behavior of the sperms compared to control. The analysis states that the rapid and sluggish behaviour of the sperms in abnormal conditions are good indicators of infertility in males (Fig. 2). The p value of mean t-test had the sperm motility in the range of $p<0.05$ in abnormal conditions.

Sperm morphology is the criteria for males having successful fertilization. While considering the sperm morphological characters such as normal form (NF), head defect (HD), neck defect (ND), cytoplasmic droplet (CD), tail defect (TD) in infertile males, the ND (4.62 \pm 2.71 ; normal: $20.5 \pm 5.16$ ) range was significantly very low, $C D$ (4.62 \pm 2.71 ; normal: $5.6 \pm 3.47)$ and TD $(1.1 \pm 0.87$; normal: $0.83 \pm 0.77)$ level did not show significant difference, NF $(2.05 \pm 0.99$; normal level: 6.5 \pm 2.2$)$ level too was less, but HD (76.48 \pm 7.78 ; normal:66.3 \pm 5.37$)$ level was significantly higher than normal. It is a known fact that the head is more important part of the sperm, wherein the defects are clearly seen in abnormal patients (males) (Fig. 3). The p value of mean t-test had $\mathrm{p}<0.0001,0.0050,<0.0001$, in normal samples and the abnormal patients had low $\mathrm{p}<0.05$ comparatively, which is quite significant.

Among the infertile men $19.0 \%$ had azoospermia, 56\% had asthenozoospermia, $3.56 \%$ had teratozoospermia, $8.14 \%$ had asthenoteratozoospermia, $14.25 \%$ had oligoteratozoospermia, $105.70 \%$ had oligoasthenoteratozoospermia, $11.40 \%$ had severe oligoasthenoteratozoospermia, and $11.40 \%$ had normozoospermia.

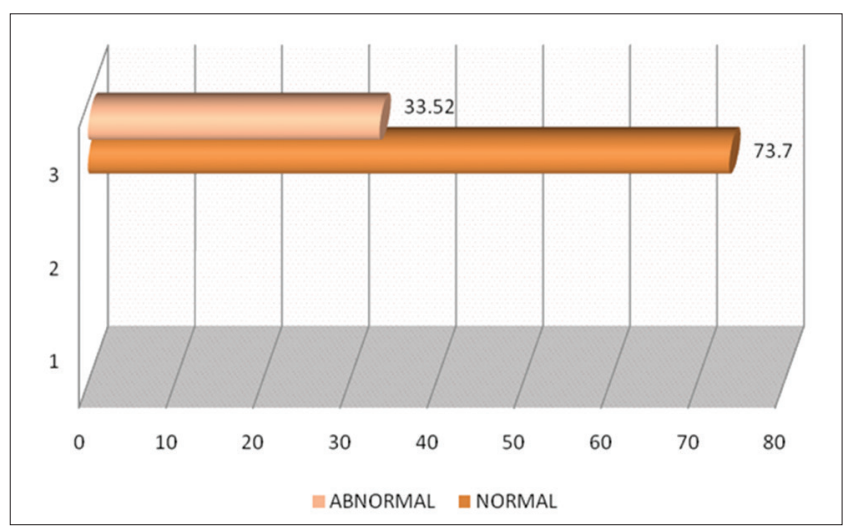

Fig. 1: Comparative diagram depicting the sperm concentration in normal and abnormal male ( $\leq 32$ age)

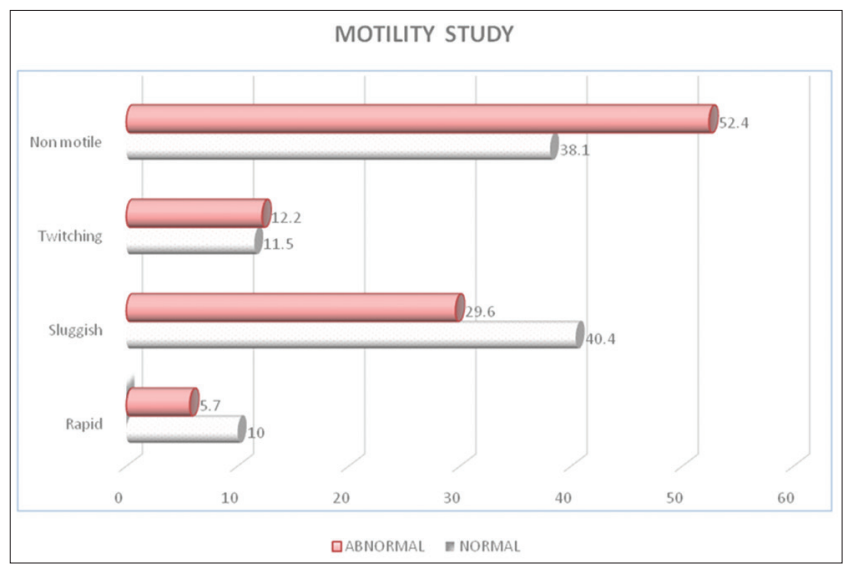

Fig. 2: Diagrammatic representation of motility test in normal and abnormal sperms in male samples
In the infertile men, higher concentration of FSH is considered to be a reliable indicator of germinal epithelial damage and was shown to be associated with azoospermia and severe oligozoospermia [15].

The GNRH such as LH, FSH, TSH, T3, T4, prolactin, and testosterone is the major contribution of this study. The results of GnRH such as T3, T4, TSH, LH, FSH, prolactin, and testosterone levels in infertile and control (proven fertile men) groups are analyzed (Table 1) and it is found that T3 (3.52 \pm 0.41 ; normal value: $3.68 \pm 0.66)$, T4 (1.33 \pm 0.14 ; normal: $1.29 \pm 0.26)$, TSH $(2.99 \pm 1.34$; normal: $2.92 \pm 1.94)$, LH $(5.93 \pm 2.31$; normal: $7.22 \pm 4.30$ ), and testosterone levels (3.27 \pm 0.85 ; normal: $3.55 \pm 1.96)$ were insignificantly conspicuous when compared to FSH (4.02 \pm 1.65 ; normal:19.64 \pm 8.89 ), and prolactin (10.45 \pm 4.73 ; normal: $13.15 \pm 11.42$ ). FSH and LH levels were significantly elevated in infertile males when compared with fertile males (control).

The impressions such as T3, T4, TSH, LH, prolactin, and testosterone had a low variation, but the FSH value was highly significant in the normal compared to abnormal values. FSH, LH, TSH, T3, T4, prolactin, and testosterone evaluation are useful in the management of male infertility [9]; FSH maintains the sperm production and the LH stimulates the production of the hormone testosterone. For initiation of spermatogenesis and maturation of spermatozoa, FSH is necessary. In the infertile men, higher concentration of FSH is considered to be a reliable indicator of germinal epithelial damage and was shown to be associated with azoospermia and severe oligoasthenoteratozoospermia [16] (Fig. 4).

The mean " $\mathrm{t}$ " test has been carried out for the all hormones (including p value) for T3, T4, TSH, FSH, LH, prolactin, and testosterone and their

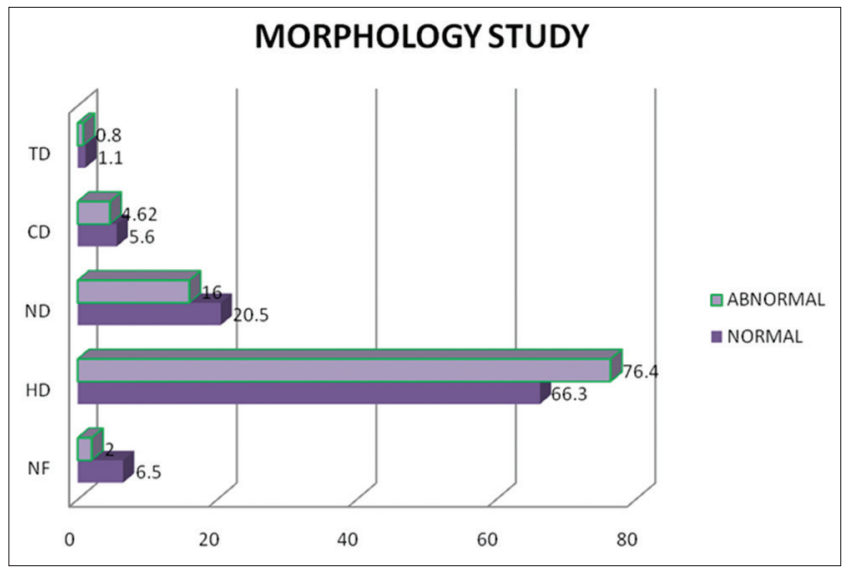

Fig. 3: Diagrammatic representation of morphological differences in normal and abnormal sperms in males

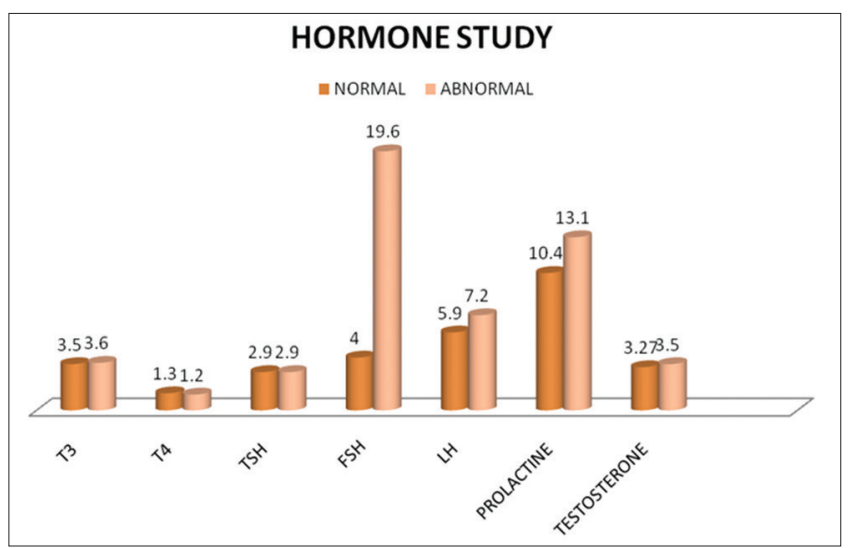

Fig. 4: Diagrammatic representation of hormonal study comparing normal and abnormal patients 
Table 1: Comparative charts depicting the various parameters adopted to characterize the normal and abnormal sperm levels in values along with their significances

\begin{tabular}{llll}
\hline Parameters & Normal & Abnormal & p value \\
\hline Age years & $29 \pm 1.88$ & $28.97 \pm 1.68$ & NS \\
Abtinance & $3.5 \pm 0.70$ & $4.36 \pm 0.31$ & NS \\
Volume (ml) & $2.05 \pm 0.76$ & $2.53 \pm 1.28$ & NS \\
pH & 8 & $7.9 \pm 0.14$ & NS \\
SP. count (M/ml) & $73.7 \pm 36.39$ & $33.52 \pm 30.50$ & $<0.0001$ \\
Motility & & & \\
Rapid (A) & $10 \pm 5.45$ & $5.73 \pm 4.90$ & $<0.0001$ \\
Sluggish (B) & $40.4 \pm 6.8$ & $29.64 \pm 14.76$ & $<0.0001$ \\
Twitching (C) & $11.5 \pm 4.6$ & $12.20 \pm 6.97$ & NS \\
Immotile (D) & $38.1 \pm 8.96$ & $52.41 \pm 17.92$ & $<0.0001$ \\
Morphology & & & \\
NF & $6.5 \pm 2.2$ & $2.05 \pm 0.99$ & $<0.0001$ \\
HD & $66.3 \pm 5.37$ & $76.48 \pm 7.78$ & 0.0050 \\
ND & $20.5 \pm 5.16$ & $4.62 \pm 2.71$ & $<0.0001$ \\
CD & $5.6 \pm 3.47$ & $4.62 \pm 2.71$ & NS \\
TD & $1.1 \pm 0.87$ & $0.83 \pm 0.77$ & NS \\
Hormone value & & & \\
T3 & $3.52 \pm 0.41$ & $3.68 \pm 0.66$ & 0.4665 \\
T4 & $1.33 \pm 0.14$ & $1.29 \pm 0.26$ & 0.6405 \\
TSH & $2.99 \pm 1.34$ & $2.92 \pm 1.94$ & 0.9142 \\
FSH & $4.02 \pm 1.65$ & $19.64 \pm 8.89$ & $<0.0001$ \\
LH & $5.93 \pm 2.31$ & $7.22 \pm 4.30$ & 0.3638 \\
Prolactin & $10.45 \pm 4.73$ & $13.15 \pm 11.42$ & 0.4686 \\
Testosterone & $3.27 \pm 0.85$ & $3.55 \pm 1.96$ & 0.6615 \\
\hline
\end{tabular}

NS: Statistically not significant, NF: Normal form, HD: Head defect, ND: Neck defect, CD: Cytoplasmic droplet, TD: Tail defect, FSH: Follicle stimulating hormone, T3: Tri-iodothyronine, T4: Thyroxin, TSH: Thyroid stimulating hormone, LH: Luteinizing hormone

levels were $0.4665,0.6405,0.9142,<0.0001,0.3638,0.4686,0.6615$, respectively. In this study, the FSH showed a significant increase $(\mathrm{p}<0.05)$ in the male young infertile groups (i.e., $>32$ year). Elevated levels of serum FSH with increasing severity of seminiferous epithelial destruction is also reported [10-12,17,18], which showed elevated levels of both FSH and LH in infertile males. Elevated levels of LH in oligozoospermia and azoospermia males when compared to normal fertile men were also reported $[9,12]$ in similar studies. The differences in the mean serum testosterone levels between fertile and infertile men were insignificant. Similar observations were made $[17,18]$. FSH, $\mathrm{LH}$, and testosterone are prime regulators of germ cell development. The quantitative production of spermatozoa generally requires the presence of FSH, LH and testosterone. FSH acts directly on the seminiferous tubules whereas LH stimulates spermatogenesis indirectly via testosterone. FSH also plays a key role in stimulating mitotic and meiotic DNA synthesis in spermatogonia [19]. This study found significantly lower total sperm counts and sperm morphologies, which contradicted with lower free testosterone level and high FSH levels which were significant.

All the subjects were normal individuals and their chromosome analysis did not present any evidence for abnormal karyotypes. In addition, there is no family history of infertility in the cases studied, and hence the infertility in these cases might be attributed to hormonal causes. The overall results clearly indicate significant increase in gonadotropins (FSH and LH) in all the subgroups (azoospermia, oligozoospermia, and varicoceles) and also in infertile males with abnormal testicular histology. Although there was no significant decrease in the testosterone levels in infertile males when compared with the fertile controls, the increase in the levels of gonadotropins might have disrupted the spermatogenic process leading to the decline in the sperm count and infertility. The importance of FSH action on sperm production has long been a matter of debate. The clinical and experimental data, thus generated in this study support a theoretical paradigm in which FSH exerts stimulatory effects on both the quantitative and qualitative aspects of testosterone driven spermatogenesis.

In conclusion, FSH may be a useful biomarker for diagnosis of infertility in young male population. Further in depth studies are required to confirm our results. More similar studies in the future may bring out a better picture related to FSH and infertility.

\section{REFERENCES}

1. Sharlip ID, Jarow JP, Belker AM, Lipshultz LI, Sigman M, Thomas AJ, et al. Best practice policies for male infertility. Fertil Steril 2002;77(5):873-82.

2. George SS, Fernandes HA, Irwin C, Chandy A, George K. Factors predicting the outcome of intracytoplasmic sperm injection for infertility. Natl Med J India 2003;16(1):13-5

3. Sigman M, Lipshultz LI, Howards SS. Evaluation of the subfertile male. In: Lipshultz LI, Howards SS, editors. Infertility in the Male. St. Louis, Missouri: Mosby-Year Book; 1997. p. 173.

4. Zargar AH, Wani AI, Masoodi SR, Laway BA, Salahuddin M. Epidemiologic and etiologic aspects of primary infertility in the Kashmir region of India. Fertil Steril 1997;68:637-43

5. Irvine DS. Epidemiology and aetiology of male infertility. Hum Reprod 1998;13 Suppl 1:33-44.

6. Dabaja AA, Schlegel PN. Medical treatment of male infertility. Trans1 Androl Urol 2014;3(1):9-16.

7. World Health Organization. Infecundity, Infertility, and Childlessness in Developing Countries. DHS Comparative Reports No 9. Calverton, Maryland, USA: ORC Macro and the World Health Organization; 2004.

8. World Health Organization. WHO Laboratory Manual for the Examination of Human Semen and Sperm Cervical Mucus Interaction. $5^{\text {th }}$ ed. Cambridge: Cambridge University Press; 2010.

9. Zabul J, Mierzejewski W, Rogoza A. Usefulness of examining gonadotropin hormones and testosterone in men with abnormal semen. Ginekol Pol 1994;65(2):71-4.

10. Sultan C, Craste de Paulet B, Audran F, Iqbal Y, Ville C. Hormonal evaluation in male infertility. Ann Biol Clin (Paris) 1985;43(1):63-6.

11. Hopkinson CR, Mauses J, Schenk B, Flitze E, Hauser CH. Some interrelationship between plasma levels of LH, FSH, oestradiol$17 \mathrm{~B}$, androgens and semen analysis data in male infertility patients. Andrologia 1977;9:216.

12. de Kretser DM. Elevated levels of serum FSH and LH with increasing severity of seminiferous epithelial damage. Clin Obstet Gynaecol 1974;1:409-27.

13. Al-Hakeim HK, Jebur IM, Alkhafaji RS. Serum anti-zona pellucida antibodies level is correlated with estradiol level in women with in vitro fertilization failure. Int J Pharm Pharm Sci 2016;8(5):41-4.

14. Sardjono RK, Pradipta V, Musthapa I. The effect of Indonesian velvet bean extract on the fertility of albino male mice. Int J Pharm Pharm Sci 2016;8(8):103-6.

15. Bergmann M, Behre HM, Nieschlag E. Serum FSH and testicular morphology in male infertility. Clin Endocrinol (Oxf) 1994;40(1):133-6.

16. Yaman O, Ozdiler E, Seçkiner I, Gögüs O. Significance of serum FSH levels and testicular morphology in infertile males. Int Urol Nephrol 1999;31(4):519-23.

17. Weinbauer GF, Nieschlag E. Gonadotropin control of testicular germ cell development. Adv Exp Med Biol 1995;317:55-65.

18. Subhan F, Tahir F, Ahmad R, Khan ZD. Oligospermia and its relation with hormonal profile. J Pak Med Assoc 1995;45(9):246-7.

19. Anderson RA, Wallace EM, Groome NP, Bellis AJ, Wu FC. Physiological relationships between inhibin $\mathrm{B}$, follicle stimulating hormone secretion and spermatogenesis in normal men and response to gonadotrophin suppression by exogenous testosterone. Hum Reprod 1997; $12: 746-7$. 